Proceedings of the European Conference Physics of Magnetism 2011 (PM'11), Poznań, June 27-July 1, 2011

\title{
The Fock Term in the Charge Ordered Phase of the $t-W$ Model
}

\author{
M. BAK \\ Faculty of Physics, A. Mickiewicz University, Umultowska 85, 61-614 Poznań, Poland \\ The paper shows the importance of retaining Hartree and Fock terms, appearing after mean-field decoupling, \\ in the treatment of charge ordered phase of the $t-W$ model, with implications to extended Hubbard model.
}

PACS: 71.45.Lr, 71.10.Fd, 71.27.+a, 71.30.+h

\section{Introduction}

Charge order in condensed matter is subject of current research in connection with high-temperature superconductors [1], manganites [2], organic conductors [3] and other materials [4]. Sophisticated methods can be used in the investigations yet the starting point of research is very often mean-field approximation (MFA). It consists in treating the operators as composed of averages plus the fluctuations, and then omitting the products of fluctuations (considered to be small). The averaged operators may be chosen in many ways from exact, multi-operator products of second quantization creation and annihilation operators. We may keep the orderings we want to examine and omit the non-interesting ones. In particular, in the Hubbard model and its extensions, which are most often used in the research of previously mentioned materials, so called Hartree (chemical potential shift) and Fock (average kinetic energy) terms arise [5]. As they appear in all phases (including normal one), even if no special ordering is considered, they can be regarded as basic (to the extent, that even the term MFA is often used interchangeably with the term "Hartree-Fock approximation" - HFA). Nevertheless, those very terms are often omitted from the calculations, especially the Fock term [5-7]. Despite there are arguments justifying such a procedure [5], inclusion of those terms is a first step towards more accurate solutions and going beyond MFA. The present paper shows the results of (broken symmetry) HFA, including both kinds of terms, applied to charge order (CDW) phase of $t-W$ model (but with some results applicable also to $t-U-W$ model) compared to the well-known results obtained without Fock term [5].

\section{Formulae}

We start with the extended Hubbard Hamiltonian

$$
\begin{aligned}
H & =-t \sum_{\langle i, j\rangle, \sigma} c_{i \sigma}^{\dagger} c_{j \sigma}-\mu \sum_{i \sigma} n_{i \sigma} \\
& +U \sum_{i} n_{i \uparrow} n_{i \downarrow}+\frac{W}{2} \sum_{\langle i, j\rangle} n_{i} n_{j}
\end{aligned}
$$

with summation $\langle i, j\rangle$ over nearest neighbors. MFA is applied to this Hamiltonian, obtaining, among other things, the Hartree terms: $n_{i \sigma} n_{j \sigma^{\prime}} \rightarrow\left\langle n_{i \sigma}\right\rangle n_{j \sigma^{\prime}}+n_{i \sigma}\left\langle n_{j \sigma^{\prime}}\right\rangle-$ $\left\langle n_{i \sigma}\right\rangle\left\langle n_{j \sigma^{\prime}}\right\rangle$, where $\langle$.$\rangle denote thermal averages with re-$ spect to the linearized Hamiltonian (which will be later calculated self-consistently). Another ordering of operators (retaining $\sigma=\sigma^{\prime}$ averages), in Gorkov-type linearization procedure, yields Fock terms ( $p$-terms): $n_{i \sigma} n_{j \sigma^{\prime}} \rightarrow c_{i \sigma}^{\dagger} c_{j \sigma} c_{j \sigma}^{\dagger} c_{i \sigma} \rightarrow\left\langle c_{i \sigma}^{\dagger} c_{j \sigma}\right\rangle c_{j \sigma}^{\dagger} c_{i \sigma}+$ H.c. $-\langle\rangle.\langle$.$\rangle .$ CDW order parameter is given as the difference of electron densities in the two sublattices $\mathrm{A}$ and $\mathrm{B}$ :

$$
n_{Q}=\left\langle n_{\mathrm{A}}\right\rangle-\left\langle n_{\mathrm{B}}\right\rangle=\frac{1}{N} \sum_{i, \sigma}\left\langle n_{i \sigma}\right\rangle \mathrm{e}^{\mathrm{i} \boldsymbol{Q} \boldsymbol{R}_{i}},
$$

where $\mathrm{e}^{\mathrm{i} \boldsymbol{Q} \boldsymbol{R}_{i}}=-1$ for any translation $\boldsymbol{R}$ transforming one sublattice into the other and $N$ is number of lattice sites. In the reciprocal space

$$
\begin{aligned}
H & =\sum_{k \sigma} \bar{\epsilon}_{k} c_{k \sigma}^{\dagger} c_{k \sigma}-\frac{1}{2} \sum_{k \sigma}\left(\Delta c_{k \sigma}^{\dagger} c_{k+Q \sigma}+\text { H.c. }\right) \\
& +C, \\
\bar{\epsilon}_{k} & =\widetilde{\epsilon}_{k}-\bar{\mu} \\
\tilde{\epsilon}_{k} & =\epsilon_{k}-\frac{1}{N} W \sum_{q} \gamma_{k-q}\left\langle c_{q \sigma}^{\dagger} c_{q \sigma}\right\rangle, \\
\Delta & =(-U+2 z W) n_{Q} / 2, \\
\bar{\mu} & =\mu-(U+2 z W) n / 2, \\
C & =-N(U+2 z W) \frac{n^{2}}{4}+\frac{W}{2 N} \sum_{k q \sigma} \gamma_{k-q}\left\langle n_{k \sigma}\right\rangle\left\langle n_{q \sigma}\right\rangle \\
& +\frac{-U+2 z W}{2 N} \sum_{k q \sigma}\left\langle c_{k \sigma}^{\dagger} c_{k+Q \sigma}\right\rangle\left\langle c_{q \sigma}^{\dagger} c_{q-Q \sigma}\right\rangle,
\end{aligned}
$$

and $\epsilon_{k}=-t \gamma_{k}, \gamma_{k}=\sum_{\delta} \mathrm{e}^{\mathrm{i} \boldsymbol{k} \boldsymbol{\delta}}, n=\frac{1}{N} \sum_{k \sigma}\left\langle n_{k \sigma}\right\rangle, z$ is number of nearest neighbors and $\delta$ are vectors connecting a given site to its nearest neighbors. After the simplification $\gamma_{k-q}=\gamma_{k} \gamma_{q} / z$ we can write the Fock term in the reciprocal space

$$
p=\frac{1}{N} \sum_{k} \gamma_{k}\left\langle n_{k \sigma}\right\rangle,
$$

which will bring single-particle energy into the form: $\bar{\epsilon}_{k}=\epsilon_{k}(1+p W)-\bar{\mu}$, where $W$ is in half-bandwidth units: $D=z t$. After diagonalization one can obtain the free energy

$$
\begin{aligned}
\frac{F}{N} & =(n-1) \bar{\mu}+(U+2 z W) \frac{n^{2}}{4}+\frac{W}{4} p^{2}-\frac{\Delta^{2}}{U-2 z W} \\
& -\frac{1}{\beta N} \sum_{k \alpha} \ln \left(2 \cosh \left(\frac{\beta E_{k}^{\alpha}}{2}\right)\right),
\end{aligned}
$$

(and grand canonical potential $\Omega / N=F / N-\mu n$ ), where 
$\beta=1 / k_{\mathrm{B}} T, k_{\mathrm{B}}$ the Boltzmann constant, and with quasiparticle energies

$$
E_{k}^{\alpha}=-\bar{\mu}+\alpha \sqrt{\epsilon_{k}^{2}(1+p W)^{2}+\Delta^{2}},
$$

where $\alpha= \pm$ and $U, W, \bar{\mu}, \Delta, F, \Omega, k_{\mathrm{B}} T$ (in short $T$ ) are in $D$ units. After differentiation in the saddle point one can obtain the equations for the CDW phase

$$
\begin{aligned}
& n-1=-\int_{-1}^{0} \mathrm{~d} \epsilon \rho(\epsilon)\left(\mathrm{th}^{-}+\mathrm{th}^{+}\right), \\
& \frac{2}{U-2 z W}=-\int_{-1}^{0} \mathrm{~d} \epsilon \rho(\epsilon)\left(\mathrm{th}^{-}-\mathrm{th}^{+}\right) / \kappa(\epsilon), \\
& p=-2(1+W p) \int_{-1}^{0} \mathrm{~d} \epsilon \rho(\epsilon) \epsilon^{2}\left(\mathrm{th}^{-}-\mathrm{th}^{+}\right) / \kappa(\epsilon),
\end{aligned}
$$

where we changed from the lattice sums to the energetic integrals with the density of states (DOS) $\rho(\epsilon)$ (due to the presence of the vector $Q$, the lattice sums are over the half of the Brillouin zone, which translates into the integrations from $\epsilon / D=-1$ to 0 , for symmetric DOS'es) and we defined: $\operatorname{th}^{\alpha}=\tanh \left(\beta E^{\alpha} / 2\right), E^{\alpha}=-\bar{\mu}+\alpha \kappa(\epsilon)$, $\kappa(\epsilon)=\sqrt{\widetilde{\epsilon}^{2}+\Delta^{2}}, \widetilde{\epsilon}=\epsilon(1+p W)$. Normal state (NO) equations can be obtained from these by putting $\Delta=0$. Remember that properly simplified $E^{\alpha}$ in this way yields $\widetilde{\epsilon}$ in normal phase $($ not $|\widetilde{\epsilon}|)$. Let us note that there are analytic solutions for rectangular DOS $[5,6]$ at $T=0$ (see Appendix).

\section{Results}

The following results were obtained for the rectangular DOS and $z=4$; all variables are in $D$ units.

As the form of $\widetilde{\epsilon}$ shows, the Fock term is connected with the $W$ parameter, so to better evaluate the influence of this term we will perform the calculations mostly for $U=0$ (and $W=0.25$ ).

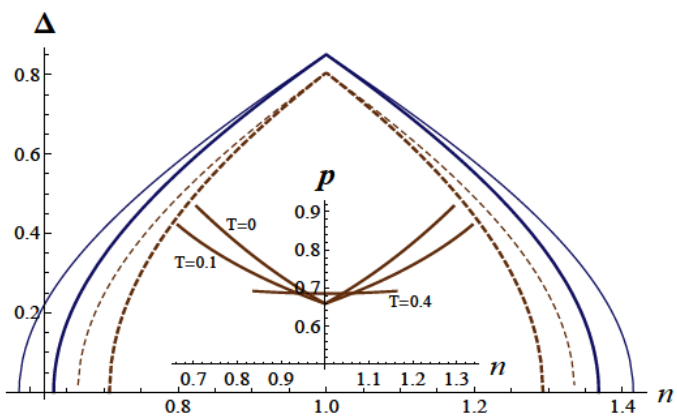

Fig. 1. CDW order parameter $\Delta$ vs. electron density $n$ of pure CDW phase, for $U=0, W / D=0.25$. Full lines - calculations without the Fock term, dashed lines including Fock term, thick lines - ground state, thin lines $-T / D=0.1$. In the inset the Fock term at various temperatures for the same values of $U$ and $W$.

In Fig. $1 \mathrm{CDW}$ order parameter is plotted versus electron density. We notice immediately that CDW phase exists only within restricted range of charge densities around half-filling, between critical densities $n_{\mathrm{c}}$ and $2-n_{\mathrm{c}}$. Increasing the temperature increases the range of existence of CDW phase (but further increase of temperature decreases CDW range), while the inclusion of the Fock term decreases both the range of existence of CDW and also its amplitude. Let us note that the above plots concern $\Delta$ in pure CDW phase which is not stabilized in lower temperatures. Below the temperature of tricritical point CDW exists only in phase separation (PS) with normal state (as will be shown later) and we can consider there "pure" CDW only within CDW fractions of phase separation or at $n=1$.

In the inset there are plots of the Fock term in three temperatures. They are to be compared with the normal state Fock term, which in the ground state for rectangular density of states is equal to $p_{\text {no }}=n(2-n)$. With increasing temperature $p_{\mathrm{cdw}}(n)$ dependence becomes more and more flat. One can also see the temperature dependence of $n_{\mathrm{c}}$ again: for larger temperatures the range of $\mathrm{CDW}$ existence shrinks.

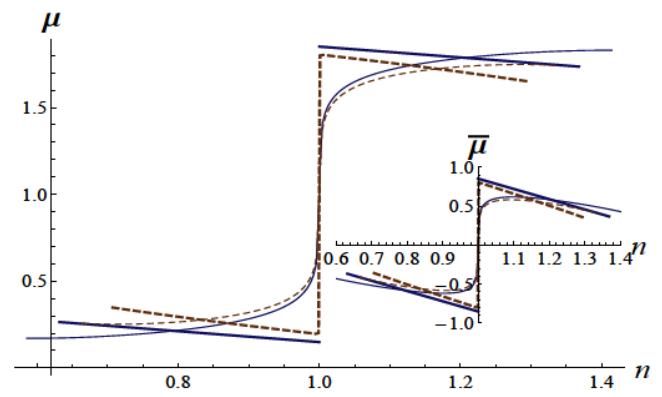

Fig. 2. Chemical potential $\mu$ and renormalized chemical potential $\bar{\mu}$ (in the inset) vs. electron density. Values of the parameters and line designations the same as in Fig. 1.

Another characteristics of the CDW phase, the chemical potential $\mu$ and renormalized chemical potential $\bar{\mu}$, are shown in Fig. 2. The plots are a bit peculiar, exhibiting a jump at $n=1$ in the ground state and sometimes negative slope vs. $n$. Negative value of $\partial \mu / \partial n$ indicates negative compressibility resulting in instability and a phase separation (PS). The actual value of $\bar{\mu}$ at half-filling is equal to zero, due to electron-hole symmetry (though in the ground state Eqs. (11)-(13) are fulfilled for any $|\bar{\mu}|<|\Delta|)$. The value of the ground state jump of $\mu$ (and also $\bar{\mu}$ ) between the values at $n=1-\varepsilon$ and $\mu$ at $n=1+\varepsilon, \varepsilon \rightarrow 0$, is equal to $2 \Delta$, reflecting the "stiffness" of the system, in which there are no "free" lattice sites to add a charge without increasing Coulomb energy (in the ground state). Only at $T>0$ thermal fluctuations disrupt the perfect CDW ordering, allowing some lattice sites to accept some additional charges. Instead of sharp jump at $n=1$ we have then a positive slope of $\mu(n)$, between some densities $n_{x}$ and $2-n_{x}$. Beyond that range the compressibility is still negative and phase separation still exists. In the ground state it is a phase separation between NO state at some density and CDW at $n=1$, 
for higher temperatures between NO and CDW with $n$ from the range between $n_{x}$ and 1 (or between $2-n_{x}$ and 1). Let us note that the behavior of $\mu(n)$ may be different from $\bar{\mu}(n)$ : the latter may show negative slope while for the same $U$ and $W$ the former may not. The phase separation is decided by the behavior of the independent variable of the grand canonical potential $\Omega$, i.e. by $\mu$.

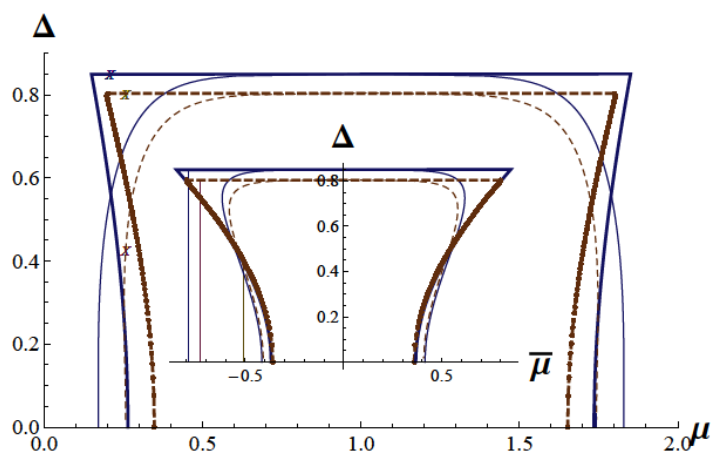

Fig. 3. CDW gap vs. chemical potential and $\bar{\mu}$ (inset). " $\mathrm{X}$ "' es (in the inset - vertical lines) show where 1st order transition happens (for $n<1$ ). Line designations the same as in Fig. 1.

Figure 3 shows the dependence of CDW gap vs. chemical potential. The negative slope of $\mu$ (or $\bar{\mu}$ ) is connected with characteristic "reentrant" behavior of $\Delta(\mu)$ (or $\Delta(\bar{\mu})$ ), which disappears when $\mu$ (or $\bar{\mu}$ ) becomes an increasing function of $n$. Let us note that points where $\Delta=0$ correspond to critical densities $n_{\mathrm{c}}$ and $2-n_{\mathrm{c}}$, while extremal values of $\mu$ (or $\bar{\mu}$ ) at which $\Delta$ still exists correspond to charge densities $n_{x}$ and $2-n_{x}$ at which $\mu(n)$ (or $\bar{\mu}(n))$ has extrema. Between $n_{x}$ (or $2-n_{x}$ ) and $n=1, \Delta$ assumes the values from the range between $\Delta\left(n_{x}\right)$ and its maximal value $\Delta(n=1)$. Values of $\Delta<\Delta\left(n_{x}\right)$ belong to metastable branch of the solution with $\partial \mu / \partial n<0$. Results including the Fock term yield the same conclusions as drawn in connection with Fig. 1.

The points where $\Delta \rightarrow 0$ are points of second order transition (II o.t.). I o.t. was found by looking for the intersection of grand canonical potentials $\Omega_{\mathrm{cdw}}(\mu)$ with $\Omega_{\text {no }}(\mu)$, both plotted vs. bare chemical potential $\mu$, Fig. 4. The $\mu$ of intersection corresponds to certain value of electron density in NO phase and different value of electron density in CDW phase (as there are two different equations connecting $\mu$ and $n$ in the two considered phases). Those $n$ 's can also be found based on plots of $F(n)$ and Maxwell construction [6], as shown in the inset in Fig. 4.

The positions of I and II o.t. for $T=0$ are shown in Fig. 5. II o.t. boundary, i.e., lines of critical density $n_{\mathrm{c}}$, are shown in the inset for temperatures $T=0$ and $T=0.1$. Increasing $T$ shrinks the area of possible CDW existence around half-filling to an exponentially small area for smaller $W$, yet for larger $W$ 's the area of CDW expands (compared to $T=0$ ). Inclusion of the

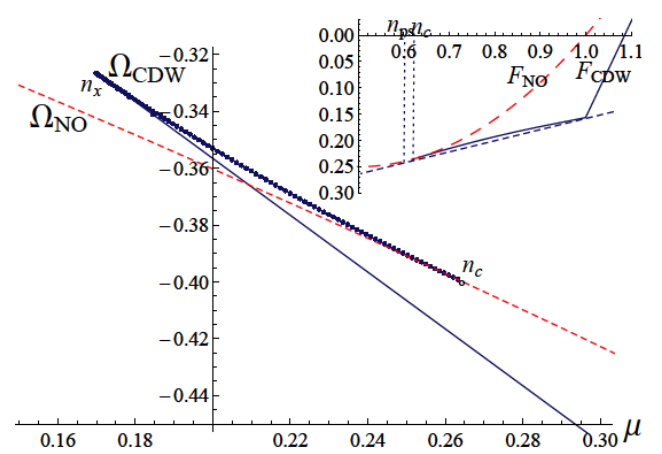

Fig. 4. Plot of $\Omega_{\mathrm{cdw}}$ (full line) and $\Omega_{\mathrm{no}}$ (dashed line) vs. $\mu$ at $T=0.1$. In the inset part of the corresponding plots of free energies $F_{\text {cdw }}$ and $F_{\text {no }}$ vs. $n$ with a dashed, straight line showing Maxwell construction; $n_{\mathrm{c}}$ shows critical density, $n_{\mathrm{ps}}$ density in the NO fraction of PS, $n_{x}$ described in the text.

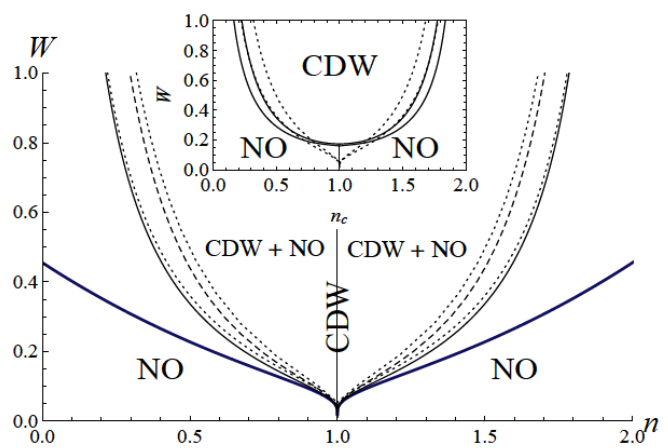

Fig. 5. Boundaries of I and II o.t. in the ground state. Thick, full, lowermost line: I o.t. boundary with no Hartree terms in (6) and (7) and no Fock (" $p$-terms"). Full line - I o.t., no $p$-term, dashed line - I o.t. with $p$-term, dotted lines closest to the full and dashed lines - curves of II o.t. without and with $p$-term. At $n=1$ there is always CDW shown by vertical line. Inset: boundaries of II o.t. for $T=0$ (dotted) and $T=0.1$ (full): external - no $p$-term, internal — with $p$-term.

Fock term into calculations always decreases the range of existence of CDW. Let us note that after mapping of $W$ into $V=U / 2-z W$ (i.e., simple renumeration of the vertical axis) the diagram is applicable not only to $t-W$ model but also to the $t-U-W$ model (as far as CDW phase is concerned): various values of $U$ and $W$, yielding the same $V$, yield the same II o.t. boundary in calculations without the Fock term (but not I o.t.). Inclusion of the Fock term breaks that "universality": for given $V$ the II o.t. boundaries calculated for $U \neq 0$ will lie between those calculated for $U=0$ (maximal influence of the Fock term) and those calculated without $p$-term.

As was shown in Ref. [8] NO-CDW transition is of I order, unlike suggestions in earlier literature [9]. Present calculations confirm that fact, even with inclusion of the Fock term. Let us note that omitting the Hartree terms: $\bar{V} n / 2$ and $-N \bar{V} n^{2} / 4, \bar{V}=U+2 z W$, from Eqs. (6) 
and (7), respectively, moves I o.t. boundary towards lower $W$ values (and is responsible for appearing of critical value of $W_{\mathrm{cr}}$, above which PS (= CDW $\left.+\mathrm{NO}\right)$ exists in the whole range of electron densities [8, 10], except $n=1$ ). Inclusion of the Hartree terms makes I o.t. boundary very close to II o.t. boundary (and removes $\left.W_{\text {cr }}[7]\right)$. Let us note also, that $n$ in PS areas is weighted average of fractions with $n_{\text {no }}$ and $n_{\mathrm{cdw}}$.

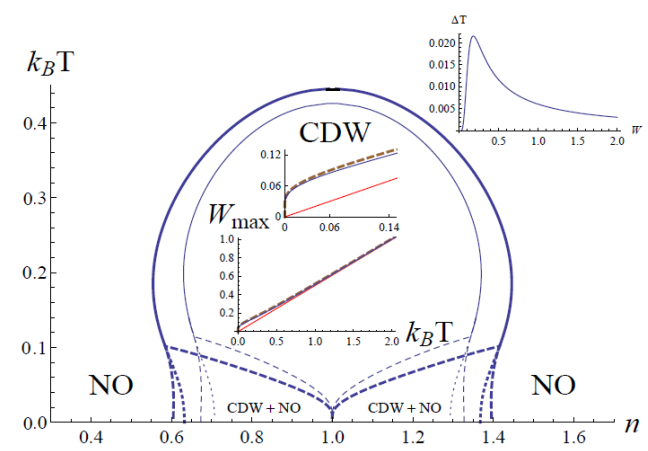

Fig. 6. Phase diagram for $U=0, W=0.25$. Thick lines - results without Fock term, thin lines - including Fock term. Bigger inset within the "dome": dependence of $W_{\max }$ (see text) on $T$ (full line - no Fock term, dashed line - with Fock term, straight line going through the point $(0,0)$ - line $W=T / 2)$, smaller inset within the "dome": enlargement of the first inset close to the axes origin. Inset outside the dome - the difference between temperatures of disappearance of CDW at $n=1$ calculated without and with the Fock term vs. $W_{\max }$.

The temperature dependence of phase diagram is shown in Fig. 6. For a given $U$ and $W$, within critical densities, for smaller temperatures there is a phase separation between NO state, with electron densities given by the external dashed lines, and CDW fractions with $n$ given by the internal dashed lines. Dotted lines within PS areas show where the II o.t. boundary would lie. At the point where the two dashed lines meet (for $T>0$ ) there are tricritical points. For larger temperatures there can be only II o.t. The maximal temperature of CDWNO II o.t. $\left(T_{\max }\right)$ appears at half-filling (at $\left.W_{\max }\right)$. $W_{\max }$ dependence on $T$ (for $U=0$ ) is shown in the inset within the "dome". Let us note that for larger temperatures $W_{\max } \rightarrow T / 2$. Inclusion of $p$-term decreases $T_{\max }$. The difference between $T_{\max }$ 's calculated without and with the Fock term is shown in the inset outside the dome. Let us note the reentrant character of the phase diagram, oblivious to the presence of the Hartree or Fock terms (yet it is absent in, e.g., molecular crystal model in $d=\infty[10])$.

\section{Discussion}

Several comments are due. The influence of the Fock term on the extended Hubbard model with $U \neq 0$ will be weaker than in described $t-W$ model.

The influence of $p$-term diminishes with increasing $z$.

The $p$-term connected with $W>0$ always destabilizes CDW, as it is connected with the increase of the absolute value of kinetic energy. After adding $U<0$ we could also consider the influence of $W<0$ on $\mathrm{CDW}$, which could be favorable (narrowing the effective bandwidth), if only we would not allow other types of ordering, like superconductivity, to set in.

The similar remark concerns large PS areas in the phase diagram or " $V$-universality" of the inset in Fig. 5: the PS areas are likely to be diminished or even removed by other pairings (or incommensurate CDW), if they are only allowed $[6,8]$ and changing $U$ and $W$ parameters, keeping constant $V$, may also stabilize other pairings (e.g. AF for $U>z W[1])$, a possibility not considered here.

The present paper is at difference with calculations omitting the Hartree term from Eq. (7) and treating $\bar{\mu}$ as an effective potential [8]. The example shown in the current paper $(\partial \mu / \partial n>0$, while $\partial \bar{\mu} / \partial n<0)$ suggests that effective potential calculations yield broader range of existence of phase separation compared to present work.

The results concerning CDW phase in HFA are closely related to results concerning antiferromagnetism (AF) in HFA (e.g., [8]), as CDW order parameter (o.p.) is transformed into AF o.p. by electron-hole transformation.

As no magnetic orderings are considered, current results should also agree with the results of $t-W$ spinless model [7].

\section{Appendix}

For completeness we remind the well known results for CDW and NO phases in the ground state with rectangular DOS [5, 6] and $p$-term added: NO: $\bar{\mu}=(n-1)$, $p=n(2-n)$. CDW: (i) $|\bar{\mu}|>|\Delta|, n \neq 1: \bar{\mu}(\bar{n})=\operatorname{sgn}(1-$ $n)[1-|n-1| \cosh (1 / \underline{V})] / \sinh (1 / \underline{V}), \underline{\Delta}^{2}=\bar{\mu}^{2}-(n-1)^{2}$, (ii) $|\bar{\mu}|<|\Delta|, n=1: \underline{\Delta}=1 / \sinh (1 / \underline{V}) ; V \equiv U / 2-z W$, $\underline{X}=X /(1+p W)$, for $X=\bar{\mu}, \Delta, V$.

\section{Acknowledgments}

The author thanks Prof. R. Micnas and Dr. P. Grzybowski for fruitful discussions.

\section{References}

[1] R. Micnas, J. Ranninger, S. Robaszkiewicz, Rev. Mod. Phys. 62, 113 (1990).

[2] E. Dagotto, T. Hotta, A. Moreo, Phys. Rep. 344, 1 (2001).

[3] T. Takahashi, Y. Nogami, K. Yakushi, J. Phys. Soc. Jpn. 75, 51008 (2006).

[4] T. Goto, B. Luthi, Adv. Phys. 52, 67 (2003).

[5] S. Robaszkiewicz, R. Micnas, K. Chao, Phys. Rev. B 24, 4018 (1981).

[6] S. Robaszkiewicz, G. Pawłowski, Acta Phys. Pol. A 90, 569 (1996).

[7] W. Czart, S. Robaszkiewicz, B. Tobijaszewska, Acta Phys. Pol. A 114, 129 (2008).

[8] B. Tobijaszewska, Ph.D. Thesis, Poznań 2001.

[9] D. Penn, Phys. Rev. 142, 350 (1966).

[10] W. Czart, P. Grzybowski, S. Robaszkiewicz, Acta Phys. Pol. A 118, 369 (2010). 